\title{
Broadband Low Reflection Surfaces with Silicon Nano-pillar Square Arrays for Energy Harvesting
}

\author{
Turgut Tut
}

\begin{abstract}
In this work, optimization of the nanopillar arrays and thin films coated on silicon substrate has been investigated in order to minimize the optical reflection loss from the silicon substrate surface. Nano-pillars' height, incline angle, array properties are systematically optimized. Full field Finite Difference Time Domain method is used to simulate EM fields and calculate the reflection loss from the modified nanostructured substrate surfaces in $400 \mathrm{~nm}-1100 \mathrm{~nm}$ spectral range. Simulation optimization recipe for the nanopillar structure is clearly presented and it is not only useful for square arrays but for regular arrays of nano-pillars in general.

Index Terms-Nano-pillars, quantum efficiency, anti-reflection, thin films, nanostructures, truncated nano-cones, solar cells, solar energy.
\end{abstract}

\section{INTRODUCTION}

G ENERALLY CRYSTALLINE silicon based photonic devices such as photodiodes, solar cells, phototransistors need low surface reflectance over a wide spectrum of light in order to have high external quantum efficiency and energy harvesting through outer circuits. One of the conventional methods to reduce surface reflection is to use single layer dielectric at specific wavelength but this does not reduce reflectivity for broadband. $\mathrm{SiO}_{2}, \mathrm{SiN}_{\mathrm{x}}, \mathrm{TiO}_{2}, \mathrm{Al}_{2} \mathrm{O}_{3}$ dielectric materials are the most popular thin film materials for antireflection coatings. When double dielectric layers are used for this purpose, there are two reflection minimums in the reflection spectrum. Unfortunately, in order to achieve a broadband low reflection, multi-layer band pass filter should be used, but this requires many thin film layers, and this increases the production costs. However, this is not an optimum solution to the broadband low reflection problem. Surface modification is needed in order to obtain broadband low reflection from the optoelectronic substrate surfaces. This modification can be achieved by using micro [1] or nano-size [2-12] structures over the device surfaces. In order to build such small features, researchers used mainly wet etching and dry etching methods. For example, industrial pyramidal surface texturing for crystalline silicon uses anisotropic wet-

TURGUT TUT, is with Department of Nanotechnology Engineering, Abdullah Gul University, Kayseri, Turkey,(e-mail: turgut.tut@ agu.edu.tr ).

iD https://orcid.org/0000-0002-4589-201X etching method. In other methods, dry etching can be used with ionized gases in the plasma vacuum chambers. For some studies both etching methods are used in combination.

Some researchers studied some degree of randomness [9$13]$ in the periodic structures to decrease the reflection further. However, for large area applications this can have some advantages as well as disadvantages as to have some standard way of production.

Structure sizes show differences in these applications. Some applications use micrometer size pillars [1] and some applications use nano-meter size pillars. For applications that need thin silicon film layers, nanometer size structures have to be used in order to achieve low reflection in broadband. If the pillars are tall (more than micron) then this can lead to quantum efficiency loss due to charge recombination in the pillar structure and the generated electron hole pairs cannot be extracted from the devices for energy harvesting. Therefore, the height of the pillars should be optimized to get low reflectivity. When the height of the pillars is too short then the coupling of incident light into the silicon substrate is low.

To summarize, recently several types of surface nanostructures have been investigated. Moth-eye nanopyramidal pillars which use graded index refractive index to reduce reflection. Nanowires and nanocylinders that use Mie resonances (scattering) which result in overall decrease in reflection. Metallic nanoparticles use their plasmonic effect to direct electromagnetic energy through the substrate effectively and decrease the reflection from the surface.

Optimization studies of the nanostructured surfaces in optoelectronic devices are still popular since the energy sector is very crucial for the economy of the countries. Even one percent increase in energy efficiency in solar cells means millions of dollars saving in countries' economies. In order to obtain high efficiency in solar cells, low reflection from the surfaces irrespective of incident angles in wide wavelength spectrum is needed.

In this work, we optimized nano-pillars filling ratio, pillar height, apex angle, and calculated the weighted average reflection with respect to wavelength to get the overall efficiency improvement for the optoelectronic devices. We focused on nano-pillar arrays of square type. 


\section{METHODS AND SiMULATION RESUlTS}

In this letter, we present the optimization of the nano-pillar square arrays in order to achieve broadband low reflection surfaces. When compared to a bare planar crystalline silicon wafer surface, the modified surface has significant reduction in reflection in $400 \mathrm{~nm}-1100 \mathrm{~nm}$ range.

We used Finite Difference Time Domain (FDTD) method to simulate photonic nanostructures. We placed monitors just below the nano-pillars to calculate the transmission to the bulk substrate. We also placed a monitor above the structure to simulate the EM fields and calculate the reflected power. We also calculate the light absorbed in the nanostructure. The optical constants of crystalline structure and thin films are taken from [14]. We first optimized the filling ratio of the nano-pillar arrays, then height to minimize the reflection. We also varied the pillar wall angle to create truncated cones to search the effect of cone structure to the reflection properties. We used dielectric anti-reflection coatings with $\mathrm{SiO}_{2}$ thin film to decrease the reflection further. These nano-cone truncated pillar structures can be fabricated using e-beam lithography and dry etching techniques. Due to the nature of etching processes, the vertical pillars can be obtained under special physical and chemical conditions $[17,18]$. It is important to have vertical pillars to get optimum reflection from such structures since as the pillar angle gets lower, the reflection start to increase Fig.7. For large area applications, nanoimprint lithography can be used with a previously prepared template using nano-fabrication methods. We also realized that to increase the efficiency of photonic devices, we have to increase the optical power absorbed in the bulk of the active silicon layer. The absorbed light in the nanostructures part could be lost due to recombination and cannot contribute to the photocurrent with large percentage. Therefore, we have to maximize the absorption not in the nanostructures but the optical absorption in the bulk silicon active device region.

Solar irradiance differs with wavelength. We can use the total reflection of light from the surface weighted with solar irradiance. This way the performance of the low reflection loss performance can be quantified better. ASTM Air Mass 1.5 direct solar irradiance is used for the calculation. In this formulation, irradiance is multiplied with the wavelength and the integral is taken with wavelength. The numerator is composed of Irradiance multiplied with wavelength and reflection. For a random polarization condition, both TE and TM polarizations are calculated, and the average is taken to get more realistic result. We used the formula given in Equation (1) in calculating the weighted averaged reflection in $400 \mathrm{~nm}-1100 \mathrm{~nm}$ wavelength range.

The novelty of our study is the following: 1) Instead of sweeping mode of the simulation, we optimized the parameters in sequence and each time, the optimization is done with respect to one parameter. This saves computational time. 2) We checked whether the angle of incline effects the reflection properties. 3) The optimization is done in terms of filling ratio, height, pillar angle, and dielectric anti-reflection coating thickness. 4) We obtained the lowest averaged reflection loss for regular square array of silicon nanopillars.

As a first step, we fixed the diameter of the pillars as $\mathbf{d}=190 \mathrm{~nm}$ and pillar height as $\mathbf{h}=120 \mathrm{~nm}$ and varied the ratio of pillar diameter to period of pillar a which is $\mathbf{d} / \mathbf{a}$ and varied that from 0.4 to 1.0. Minimum weighted averaged reflection is obtained as 3.91 percent at $\mathbf{d} / \mathbf{a}=0.7$ as depicted in Fig. 2. We used a to denote the period of the square nano-pillar array. Since we are dealing with the thin film device applications, we take the height range $60 \mathrm{~nm}-190 \mathrm{~nm}$ since larger and smaller height nano-pillar arrays have high reflectivity in the interested spectral region. As depicted in Fig.3, and Fig.4, we varied the height of the pillars and we found that $120 \mathrm{~nm}$ is the optimum height especially for low reflection in $400 \mathrm{~nm}$ $1100 \mathrm{~nm}$ range. For large pillar heights, the reflectivity increases especially for UV region in the spectrum. For shorter pillars, the reflection increases especially for wavelengths larger than $600 \mathrm{~nm}$. The weighted averaged reflection calculations for the whole set of height range can be seen in Fig.4. Therefore, we fixed the pillar height at $120 \mathrm{~nm}$. (a)

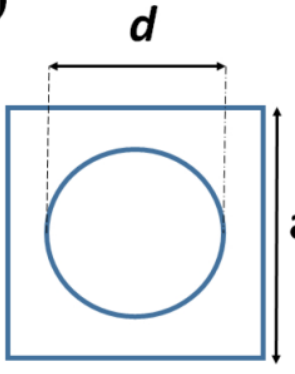

Fig.1. (a) Unit cell used in the simulation of square nanopillars, (b) Cross section of truncated pillar structure to define the pillar angle (b)

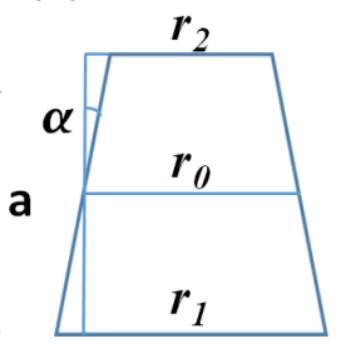

Weighted Average Reflection $=\frac{\int_{400}^{1100} \frac{R_{T E}(\lambda)+R_{T M}(\lambda)}{2} I(\lambda) \lambda d \lambda}{\int_{400}^{1100} I(\lambda) \lambda d \lambda}$

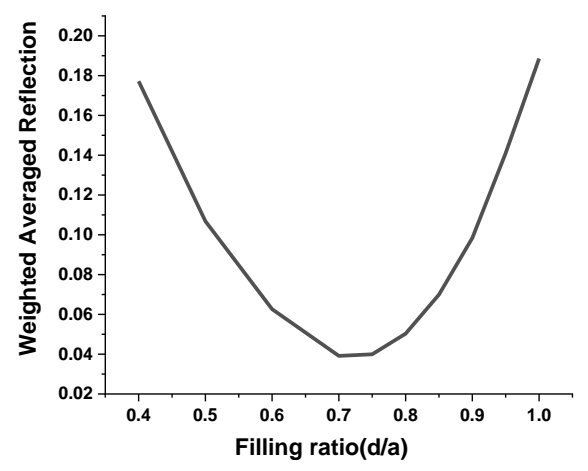

Fig.2. Weighted averaged power versus filling ratio of the pillars 


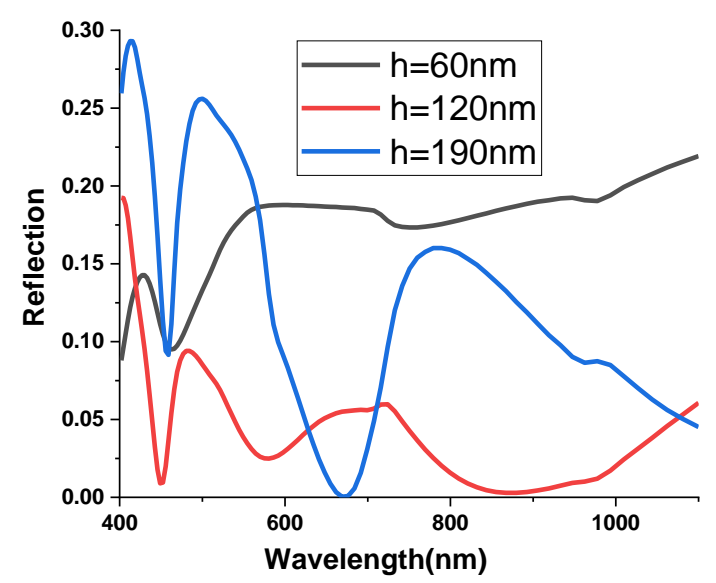

Fig.3. Height optimization of the pillars

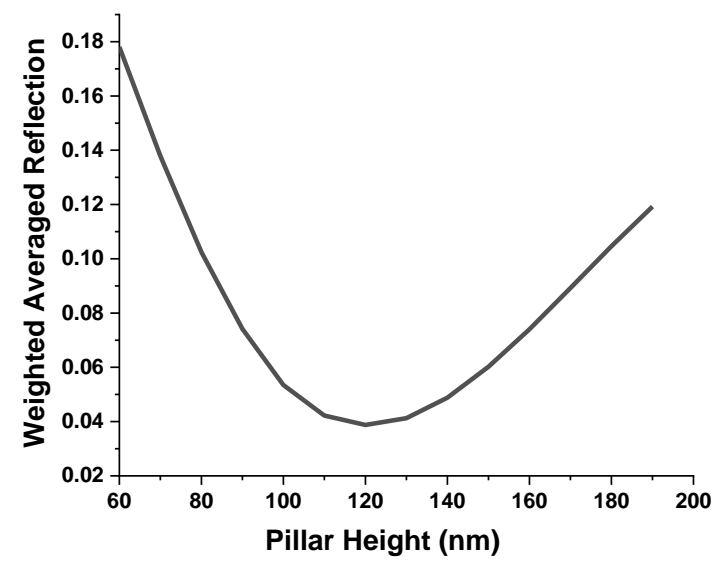

Fig.4. Weighted Averaged Reflection versus Pillar height

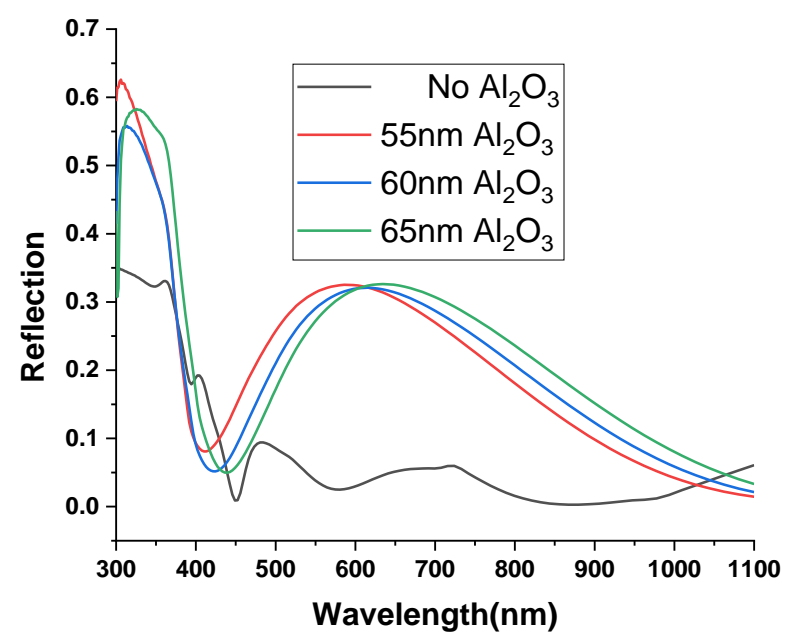

Fig.5. Anti-reflection dielectric $\mathrm{Al}_{2} \mathrm{O}_{3}$ thin film coating on the nanopillar array

We further examine the anti-reflection dielectric film $\mathrm{Al}_{2} \mathrm{O}_{3}$ coating. In figure $5,55 \mathrm{~nm}, 60 \mathrm{~nm}$, and $65 \mathrm{~nm}$ thick $\mathrm{Al}_{2} \mathrm{O}_{3}$ thin film added at top of the nano-pillar structures. As can be noticed, the reflection is minimum and small in about $50 \mathrm{~nm}$ range around the minimum [15]. At other spectral wavelength range $400 \mathrm{~nm}-1100 \mathrm{~nm}$, the reflection is even larger than we obtained when only nano pillar arrays are used Fig.5.
Therefore, instead of using the concept of antireflection minimization at one point, the analysis of a range of thin film thickness is needed using FDTD simulations.

As depicted in Fig.1(b), the truncated cone architecture is used to decrease furher the reflection from the surface. The angle at which the pillar walls make with the vertical varied between 0-26 degrees. As can be seen in Fig.6, for wavelengths below $550 \mathrm{~nm}$, the reflection decreases with pillar slope however above $550 \mathrm{~nm}$, the reflection increases considerably. Truncated nanopillars apex angle can be achieved with optimizing the dry etching conditions, e.g., chamber pressure, temperature, gas flow rates. When the weighted average reflection is calculated for different pillar slopes (delta $r$ is the difference of the bottom and top truncated nanocone pillar diameters) as shown in Fig.7. The minimum weigted averaged reflection occures for vertical pillars therefore it is very important to obtain vertical pillars in square nanopillar arrays to obtain minimum reflection surfaces. For wavelengths smaller than 550nm, truncated nanocone arrays have lower reflections. However, for wavelengths larger than $550 \mathrm{~nm}$, they have higher reflections. For specific range of wavelengths, this property can be useful.

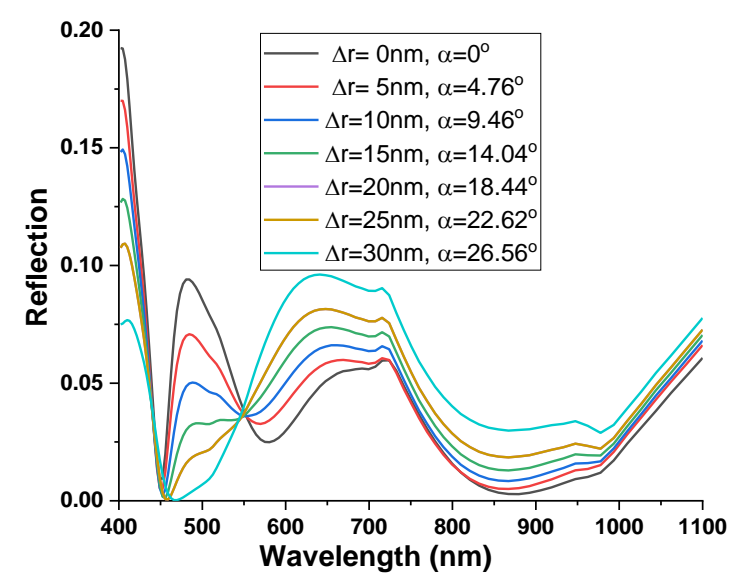

Fig.6. Reflection optimization with cone angle alpha.

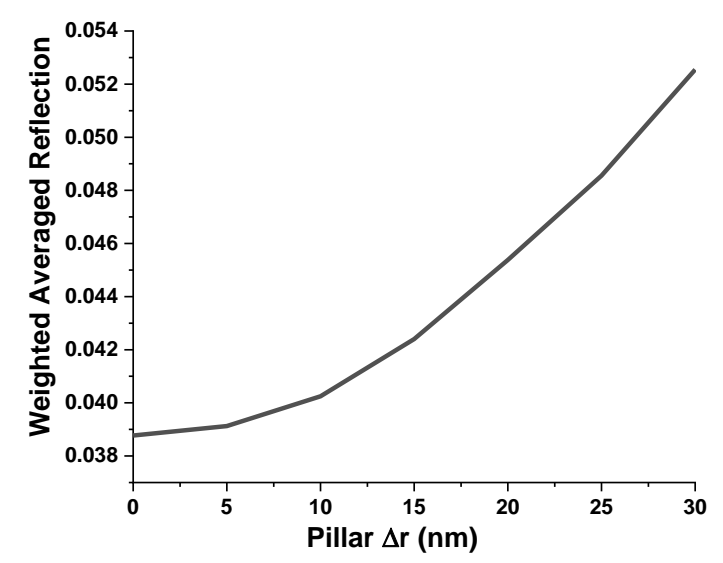

Fig.7. Total weighted reflection with solar irradiance on earth surface versus pillar angle. 


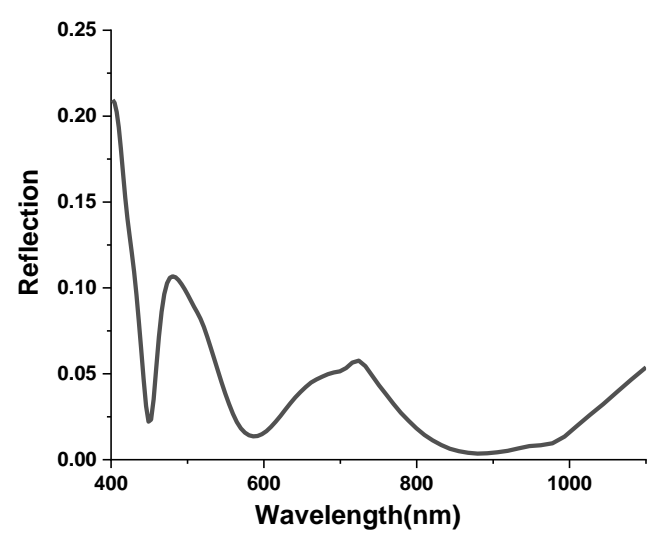

Fig.8. The optimum structure reflection versus wavelength with $5 \mathrm{~nm} \mathrm{SiO}_{2}$ thin film.

It is also noticeable that the reflection is relatively high for the UV spectral region below 450nm wavelength. This is due to the fact that at small wavelengths the light rays start to ignore the nano-pillar array structure and get reflected from the bare silicon substrate surface. Therefore, reflection loss is high for UV region. Dielectric anti-reflection coating is also investigated by depositing $\mathrm{SiO}_{2}$ layer on top of the all structure with thicknesses varied from $5 \mathrm{~nm}$ to $70 \mathrm{~nm}$. The minimum reflection is obtained with weighted averaged reflection of 3.75 percent with $5 \mathrm{~nm}$ thickness in $400 \mathrm{~nm}$ $100 \mathrm{~nm}$ range which is one of the lowest reflection losses reported in the literature. The reflection spectra is depicted in Fig.8. When we compare the recent results from other groups, the lowest value for the average reflection from regular nanopillar square arrays is $4 \%$ Table 1 . This averaged reflection loss can be avoided further if compound nanopillars with a scattering tip on top of the pillar are used so that light can be absorbed in the substrate not in the pillar structures. The absorption in the UV region of the spectrum at the nano-pillar array structure is large; this is also a big issue if there is recombination in the nanostructure for energy extraction from an active device. We will pursue these issues in future work.

TABLE I

COMPARISON OF RECENT RESULTS FOR THE AVERAGE REFLECTIONS FROM REGULAR SQUARE NANOPILLAR ARRAYS

\begin{tabular}{|lc|l|c|}
\hline Author & & Year & R (Average) \\
\hline J. Li et al. & {$[19]$} & 2009 & $>10 \%$ \\
\hline C. Lin et al. & {$[20]$} & 2011 & $4.64 \%$ \\
\hline J. Proust et al. & {$[21]$} & 2016 & $4 \%$ \\
\hline J. Kim et al. & {$[22]$} & 2021 & $7.1 \%$ \\
\hline
\end{tabular}

\section{CONCLUSION}

The reflection properties of the nanostructured silicon surfaces have been investigated and understood physically through weighted average reflection as a function of wavelength. We see that using truncated nano-cones has potential to reduce further the total weighted reflection loss from the surface of the photonic devices surfaces for specific wavelength range. For broadband low reflection, vertical nano-pillars should be used for square pillar arrays. We developed a simulation recipe to achieve minimum weighted average reflection architecture of the structure with respect to pillar height, filling ratio, incline angle, dielectric anti-reflection coating. Depositing $5 \mathrm{~nm} \mathrm{SiO}_{2}$ thin film, one of the minimum reflections is obtained for square array type nano-pillar structures in the literature, which is $3.75 \%$. In order to optimize nanopillar structure further, adding a scattering tip structure on top of the pillars will be investigated as future study.

These modified nanostructured surfaces concept is useful for photonic device applications having thin film silicon or active absorbing semiconducting material for optical energy harvesting through low surface reflection over a broadband optical spectrum [16]. In broad sense, thin film solar cells, photodetectors, phototransistors applications are the potential applications of these nanostructured surfaces with low reflection.

\section{ACKNOWLEDGMENT}

This work is funded by TUBITAK 1001 project under project number 219M280. Computing resources were supported by AGÜ (Abdullah Gul University). I would like to thank my colleagues Evren Mutlugün and Mehmet Şahin for valuable discussions on the subject.

\section{REFERENCES}

[1] P. Campbell, M A. Green. "Light trapping properties of pyramidally textured surfaces." J. Appl. Phys. Vol. 62. No.1, 1987, pp 243-249.

[2] S. Chattopadhyay, Y.F. Huang, Y.J. Jen, A. Ganguly, K.H. Chen, L.C. Chen. "Anti-reflecting and photonic nanostructures." Mater. Sci. Eng. Rep., Vol.69. No.1-3, 2010, pp 1-35.

[3] P. Lalanne and G. M. Morris, "Antireflection behavior of silicon subwavelength periodic structures for visible light." Nanotechnology Vol. 8. No.2, 1997, pp 53-56.

[4] Y. Kanamori, M. Sasaki, and K. Hane, "Broadband antireflection gratings fabricated upon silicon substrates." Opt. Lett. Vol.24. No.20, 1999, pp 1422-1424.

[5] K. Hadobás, S. Kirsch, A. Carl, M. Acet, and E. F. Wassermann, "Reflection properties of nanostructure-arrayed silicon surfaces." Nanotechnology Vol.11. No.3, 2000, pp 161-164.

[6] H. Sai, H. Fujii, K. Arafune, Y. Ohshita, M. Yamaguchi, Y. Kanamori, and H. Yugami, "Antireflective subwavelength structures on crystalline Si fabricated using directly formed anodic porous alumina masks." Appl. Phys. Lett. Vol.88, No.20, 2006, pp 201116.

[7] S. A. Boden and D. M. Bagnall, "Tunable reflection minima of nanostructured antireflective surfaces." Appl. Phys. Lett. Vol.93. No. 13, 2008, pp 133108.

[8] Y.-H. Pai, Y.-C. Lin, J.-L. Tsai, and G.-R. Lin, "Nonlinear dependence between the surface reflectance and the duty-cycle of semiconductor nanorod array." Opt. Express Vol.19. No. 3, 2011, pp 1680-1690.

[9] Y.-F. Huang, S. Chattopadhyay, Y.-J. Jen, C.-Y. Peng, T.-A. Liu, Y.-K. Hsu, C.-L. Pan, H.-C. Lo, C.-H. Hsu, Y.-H. Chang, C.-S. Lee, K.-H. Chen, and L.-C. Chen, "Improved broadband and quasi-omnidirectional anti-reflection properties with biomimetic silicon nanostructures." Nat. Nanotechnol. Vol.2. No.12, 2007, pp 770-774.

[10] H. Sai, Y. Kanamori, K. Arafune, Y. Ohshita, and M. Yamaguchi, "Light trapping effect of submicron surface textures in crystalline $\mathrm{Si}$ solar cells.” Prog. Photovolt. Res. Appl. Vol.15. No.5, 2007, pp 415423.

[11] S. A. Boden and D. M. Bagnall, "Optimization of moth-eye antireflection schemes for silicon solar cells." Prog. Photovolt. Res. Appl. Vol.18. No.3, 2010, pp 195-203. 
[12] H. Sai, H. Fujii, K. Arafune, Y. Ohshita, Y. Kanamori, H. Yugami, and M. Yamaguchi, "Wide-angle antireflection effect of subwavelength structures for solar cells.” Jpn. J. Appl. Phys. Vol. No. 46-6A, 2007, pp 3333-3336.

[13] P. Seliger, M. Mahvash, C. Wang, and A. F. J. Levi, “Optimization of aperiodic dielectric structures.” J. Appl. Phys. Vol. 100. No.3, 2006, pp 034310-034316.

[14] D. F. Edwards, "Silicon (Si)," Handbook of Optical Constants of Solids, E.D.Palik, ed. (Academic, Orlando, Fla., (1985).

[15] B. L. Sopori and R. A. Pryor, "Design of antireflection coatings for textured silicon solar cells." Sol. Cells Vol.8. No.3, 1983, pp 249-261.

[16] D. Shir, J. Yoon, D. Chanda, J.-H. Ryu, and J. A. Rogers, "Performance of ultrathin silicon solar microcells with nanostructures of relief formed by soft imprint lithography for broad band absorption enhancement." Nano Lett. Vol.10. No.8, 2010, pp 3041-3046.

[17] H. Park, Y. Dan, K. Seo, Y. J. Yu, P. K. Duane, M. Wober, and K. B. Crozier, "Filter-Free Image Sensor Pixels Comprising Silicon Nanowires with Selective Color Absorption." Nano Lett. Vol.14. No.4, 2014, pp 1804-1809.

[18] T. Tut, Y. Dan, P. Duane, Y. Yu, M. Wober,and K. B. Crozier, "Vertical waveguides integrated with silicon photodetectors: Towards high efficiency and low cross-talk image sensors.", Appl. Phys. Lett. Vol.100., 2012, pp 043504.

[19] J. Li, H.u Yu, S. M. Wong, G. Zhang, X. Sun, P. G. Lo, and D. Kwong, "Si nanopillar array optimization on Si thin films for solar energy harvesting.", Appl. Phys. Lett. Vol. 95, 2009, pp 033102.

[20] C. Lin, N. Huang, and M. L. Povinelli, "Effect of aperiodicity on the broadband reflection of silicon nanorod structures for photovoltaics.", Opt. Express Vol. 20, No.1, 2011, pp 125-132.

[21] J. Proust, A. Fehrembach, F. Bedu, I. Ozerov, N. Bonod, “Optimized 2D array of thin silicon pillars for efficient antireflective coatings in the visible spectrum.”, Sci. Rep. Vol. 6, 2016, pp 24947.

[22] J. Kim, S. You, C.Kim, "Surface Texturing of Si with Periodically Arrayed Oblique Nanopillars to Achieve Antireflection.”, Materials Vol. 14, 2021, pp380.

\section{BIOGRAPHIES}

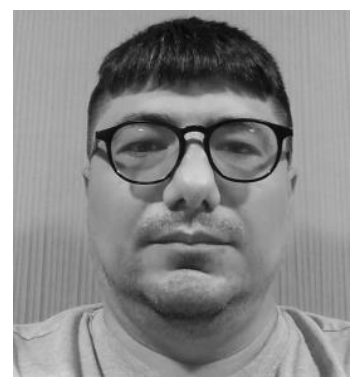

TURGUT TUT was born in Mersin, Turkey, in 1978. He received his B.S. and M.S. degrees in physics from Bilkent University, in Ankara, Turkey, in 2004 and the Ph.D. degree in physics from Bilkent University, in Turkey, in 2008. From 2008 to 2011, he was a Postdoc researcher at Harvard University. He has been working at Abdullah Gül University as Assistant Professor since 2018. He is the author of more than 20 articles, and one patent. His research interests include micro/nano-pillars, photodetectors, micro/nano-lithography techniques, thin film transistors, nanophotonics, numerical simulations of quantum systems and photonic devices. 